\title{
Atrial Septal Defect Device Closure in Patients with Metabolic Diseases or Genetic Syndromes
}

\author{
Keyhan Sayadpour Zanjani ${ }^{1}$, Morteza Heidari ${ }^{1}$, Nima Nazari ${ }^{1}$, and Reihaneh Mohsenipour ${ }^{1}$ \\ ${ }^{1}$ Tehran University of Medical Sciences
}

December 9, 2021

\begin{abstract}
Concomitant metabolic or genetic syndromes can make atrial septal defect device closure difficult. We searched our database and found eight. The cohort consists of patients with different metabolic and genetic disease. Thromboembolic events and device embolization occurred in some patients. Careful attention is recommended in patients with special diseases.
\end{abstract}

\section{Introduction}

Atrial septal defect (ASD) is one of the most common congenital heart diseases. Transcatheter device closure of ASD has been introduced in 1974 by King et al (1). This therapy is now the method of choice for most defects. Lower burden of trauma to the patient, avoidance of cardiopulmonary bypass, and a better cosmetic result are some benefits of device closure over surgery (2). The risk of serious complications is very low and mortality is extremely rare in large cohorts (3). However, it may not be the same for the patients with a concomitant extracardiac disease. Metabolic disease and genetic syndromes may change the scenario towards serious complications and even death.

As our hospital is a referral center for children with complex and rare diseases from our country, we encounter these patients relatively frequently. As far as we know, there is no report in the medical literature about this subject, besides scattered case reports. In this case series, we analyzed the problems we had during ASD device closure in these kinds of patients.

\section{Methods}

The medical records of 178 ASD device closure attempts performed between September 2006 and September 2021 in our hospital by a single performer were searched for patients with either a metabolic or a genetic disease. A total of 8 patients were found and are included in this case series.

ASD closure was done as previously described $(2,4-6)$. We routinely used balloon sizing in the beginning of this period (Zanjani, Mortezaeian). Gradually, balloon sizing has been abandoned for most of the patients, relying on the size of the defect on transesophageal (TEE) or 3 dimensional transthoracic echocardiographies (Eshraghi). Data were expressed as median and range whenever applicable.

\section{Results}

The patient's data were summarized in Table 1. The median age of the patients was 4.25 years (3-14). Their median weight was $13.25 \mathrm{~kg}(4-94)$.

Two patients had impaired glucose metabolism (insulin dependent diabetes mellitus in one; metabolic syndrome with obesity, hypertriglyceridemia, and clinical symptoms of insulin resistance in the other one). 
Two patients had suspected genetic syndromes, including one with Seckel syndrome (severe growth retardation, developmental delay, facial abnormalities) and another one with Alagille syndrome (jaundice, bile duct paucity).

The procedure was successful in seven patients $(87.5 \%)$. One patient was operated on after device embolization. The median follow up duration for 6 patients (excluding one recent procedure) is 3 years $(1.5-11)$. One of the patients had an ischemic stroke in the territory of the right middle cerebral artery during the follow up period (Figure 1). Detailed data of each patient are presented below.

Table 1: Main data of the patients. DWR, device size to body weight ratio; F/U, follow up; IDDM, insulin dependent diabetes mellitus; MCA, middle cerebral artery ; ASD-R (pfm AG, Germany); Starway (Starway Cardi-O-Fix ASD occlude, Starway Medical Technology, China); Figulla (Figulla flex ASD occluder, Occlutech, Sweden); Amplatzer (Amplatzer Septal Occluder, Abbott Laboratories, USA).

\begin{tabular}{lllll}
\hline Patient & Age (years) & Weight $(\mathrm{kl})$ & Underlying disease & Complications \\
\hline 1 & 12 & 26 & IDDM & Cardiac thrombi, device embolis \\
2 & 4 & 9 & Mitochondrial disease & - \\
3 & 5 & 15 & Rickets & - \\
4 & 4 & 4 & Seckel syndrome & - \\
5 & 3 & 17 & Alagille syndrome & - \\
6 & 3 & 11 & Crigler Najjar syndrome type II & - \\
7 & 14 & 94 & Metabolic syndrome & Ischemic stroke (right MCA) \\
8 & 4.5 & 11.5 & Combined oxidative phosphorylation deficiency-35 & - \\
\hline
\end{tabular}

Patient 1 : A 12-year-old girl with large ASD and insulin dependent diabetes mellitus was referred to us for ASD device closure. Although she received insulin, the control was poor with high blood glucose and HbA1c levels (fasting blood glucose $243 \mathrm{mg} / \mathrm{dL}, \mathrm{HbA1c} 8 \%$ ). She was hospitalized and her blood glucose was normalized prior to the intervention. The defect was around $12-13 \mathrm{~mm}$ on echocardiography with acceptable rims.

In the catheterism laboratory, she received $70 \mathrm{unit} / \mathrm{kg}$ of heparin. We measured the ASD size by a $25 \mathrm{~mm}$ Occlutech sizing balloon (Occlutech, Sweden) and waist method. The defect size was $15.5 \mathrm{~mm}$. We attempted to implant a $16 \mathrm{~mm}$ ASD-R (pfm AG, Germany) but the device was easily passed through the defect. Based on our protocol in this situation, we decided to implant a device 2 size larger, i.e. $20 \mathrm{~mm}$ ASD-R. At this stage, we noticed on echocardiography that a large mass appeared on the right atrium originating from the septum between the defect and tricuspid valve. We assumed that the mass should be a thrombus. As activated clotting time (ACT) was 170 seconds; extra heparin was administered and ACT was increased to 300 seconds. We needed a larger sheath for a $20 \mathrm{~mm}$ ASD-R device and there was the risk of thrombus embolization in case of long sheath exchange. Therefore, we decided to try an $18 \mathrm{~mm}$ ASD-R. The device was implanted and was positioned acceptably.

Late afternoon, the patient developed ventricular arrhythmias in the intensive care unit. Echocardiography showed that the device was in the right ventricle. The patient was operated on the next day. There were some damages to the pulmonary and tricuspid valves which were repaired. The device and thrombus were removed and the defect was closed. The surgeon confirmed the thrombus origin from the interatrial septum. The postoperative course was favorable without any neurologic or cardiac complications. This is the only ASD device embolization in the cohort of 178 ASD device closures during the study period of 15 years.

Patient 2 : The metabolic disease of this patient was mitochondrial disease (growth retardation, developmental delay, microcephaly, brain atrophy and history of cataract surgery). High lactate levels and high anion gap acidosis were always present in her laboratory data. The procedure was done at the age of 4 years. Both her weight $(9 \mathrm{~kg})$ and device size to body weight ratio (DWR, 1.56) were slightly out of the safe zone. 
However, the procedure was done without any problem. She had a weight gain of 4 kilograms during the year after the procedure.

Patient 3: An uneventful procedure was done in a patient with vitamin D resistant rickets, receiving vitamin D3, calcium supplement and dihydrotachysterol (AT 10).

Patient 4: A patient with severe growth retardation (birth weight $700 \mathrm{gr}$, weight at 4 years of age only $4 \mathrm{~kg}$ ), microcephaly, developmental delay, facial abnormalities, and clinically highly suspected of Seckel syndrome) was followed up since infancy (Figure 2). Finally at the age of 4 years, we decided to close the defect as reaching higher weight during the following years was not anticipated. His weight $(4 \mathrm{~kg})$ and DWR (2.25) were much beyond the safe zones. However, the defect was closed successfully, and he had no problem during the next 3 years after the procedure. His weight was increased to $6 \mathrm{~kg}$ meanwhile.

Patient 5 : In a patient with Alagille syndrome (jaundice, bile duct paucity, and ASD as the only cardiovascular abnormality), the defect was closed without any problem.

Patient 6 : Although we used a device with high DWR (1.55), the ASD in this patient with CriglerNajjar type II syndrome (jaundice since neonatal period, hypothyroidism, growth retardation) was closed successfully.

Patient 7 : He was a 14 years old boy with severe obesity (body mass index $36, \mathrm{Z}$ score 4.2 ), hypertriglyceridemia, clinical symptoms of insulin resistance and impaired glucose tolerance test. After a successful ASD device closure, he was discharged with the recommendation of using clopidogrel for 3 months and aspirin for one year. He deliberately stopped the use of aspirin after 9 months. Two weeks later, left hemiparesis occurred. Magnetic resonance imaging showed large areas of diffusion restriction around the right Sylvian fissure, indicating an ischemic event in the territory of the right middle cerebral artery (Figure 1). He improved gradually thereafter.

Patient 8: In a patient with severe growth retardation, global developmental delay, history of seizure and a confirmed diagnosis of combined oxidative phosphorylation deficiency-35, ASD was closed successfully. Mitochondria play an important role in intracellular calcium metabolism and may also have a subsidiary role in the progression of malignant hyperthermia(7). Before using the anesthesia machine, the anesthesia circuit was flushed with oxygen during 2 hours. Carbon dioxide absorber was replaced before induction of anesthesia. Induction was started with midazolam and fentanyl while propofol infusion and cisatracurium were used for the maintenance. Arterial blood gas (ABG) was checked twice during the procedure (immediately after the start of anesthesia and before extubation). All ABG parameters were normal and no metabolic disturbance was observed. The patient was extubated, awakened and transferred to the intensive care unit with stable vital signs. Unfortunately, essentially every general anesthetic studied has been shown to depress mitochondrial function (8). Inhaled gases were not used to avoid malignant hyperthermia. Due to the history of seizures in her recent hospitalization, ketamine infusion was not used. Propofol infusion was finally selected for the maintenance of anesthesia. It is important to note that the durations of the procedure and anesthesia were short.

\section{Discussion}

Although this cohort consists of a small percentage of ASD closures during the study period (4\%), it includes the worst cases, i.e. the only cases of ischemic stroke and device embolization. The remaining 6 patients were also difficult in terms of their non-cardiac problems and small body size for age or defect size.

The patients with metabolic or genetic diseases are not homogenous. However, many of them share similar problems that should be considered before, during, or after ASD device closure. Some of these common problems are listed below.

\section{Patient size}

Many of these patients are interrogated at a young age for possible cardiac disease and their ASD is often discovered early. They often have growth retardation, for which both the metabolic or genetic disease and 
the ASD may be responsible. While the metabolic or genetic diseases have often no definitive or simple cure, the ASD can be easily cured. Therefore, the parents like to treat it earlier for the weight gain of their child.

ASD device closure is generally recommended in patients over $10 \mathrm{~kg}$ of weight $(9,10)$. Cardiac arrhythmias especially complete heart block, and erosion can be the risks of ASD closure in small children. However, there are reports of successful closure of ASD in smaller patients $(9,10)$. For two patients $(2$ and 4$)$, we postponed the procedure to the age of 4 years and then attempted to close the defect, in spite of a weight less than $10 \mathrm{~kg}$. Both procedures were successful without any problem.

In addition, a DWR more than 1.5 is generally regarded as a high-risk procedure (11). There are reports of safe ASD closures with devices larger than 1.5 times the patient weight (11). We had 3 patients with DWR more than 1.5 (patients 2, 4 and 6; two of them had also a weight less than $10 \mathrm{~kg}$ ). The procedure was successful in all of them. It seems that ASD device closure in these small patients does not carry a much higher risk, if postponed to at least 4 years of age.

\section{Hypercoagulation}

Hypercoagulopathy can be seen in metabolic disease, especially in diabetes mellitus and insulin resistance (12). We had two patients with these abnormalities and both have been complicated by significant thromboembolic events. Aggressive control of diabetes before the procedure, higher anticoagulation with precise control of ACT during the procedure, and longer antiplatelet therapy may be advisable.

\section{Anesthesia}

Metabolic diseases are a group of congenital diseases that cause major problems in anesthesia and surgery (13). These diseases are heterogeneous and in each of them, special anesthesia considerations should be considered. Hepatic disease, difficult intubation, risk of malignant hypertension, and renal disease can be some of the problems that anesthesiologist may face. Careful control of water and electrolyte balance, and blood sugar level is important $(14,15)$. Continuous ABG measurement can be used to monitor electrolyte disturbances and metabolic abnormalities.

Patients with mitochondrial disease are prone to high lactate levels and consequent acidemia due to prolonged fasting. Therefore, prolonged fasting should be avoided in these patients (8). In mitochondrial disorders, nearly all general anesthetics (volatile and parenteral) depress mitochondrial function (16). However, bolus doses of propofol have been advocated in some studies. Propofol infusion has not been recommended and can be used only for short time (17).

\section{Echocardiography}

Echocardiography is used generally to guide the interventionist during ASD device closure. Transesophageal echocardiography is the most accepted type. However, bleeding tendencies, esophageal or laryngeal diseases, and small size of the patient in those with the metabolic or genetic diseases may limit its use (18). Although transthoracic echocardiography is less widely accepted, it can be used safely in these cases (2).

\section{Contrast media}

Patients with genetic or metabolic disease may suffer from renal function impairment and this may be aggravated by contrast media. Fortunately, angiography has been replaced significantly by echocardiography and the use of contrast media is now limited during ASD device closure.

\section{Cardiac Failure}

Cardiac failure after ASD device closure in adults is a clinical problem. It rarely occurs in children. It can be seen in children with compensated cardiac failure and metabolic disease after ASD device closure (19).

ASD is not a known feature of Seckel syndrome(20) (Saeidi). As far as we know, our cases of Seckel syndrome is the first one with reported ASD device closure in the medical literature. ASD was reported in patients with combined oxidative phosphorylation deficiency-35 $(21,22)$. However, our patient is the first one for 
whom device closure was done, to the best of our knowledge. Successful ASD device closure in Alagille syndrome has been already reported in the medical literature (23).

\section{Limitations}

Our most important limitation was the definite diagnosis of the metabolic or genetic diseases. The availability of genetic testing in our country is limited. Most specimens should be sent abroad, which is expensive and not covered by insurance companies. The diagnosis in most cases was done based on the clinical and laboratory findings. Although the diagnosis may not be accurate, the reported results and experiences may be valuable as they have occurred most probably in the setting of a metabolic or genetic disease, regardless of the exact diagnosis. The retrospective nature of the report and lack of some data or long term follow up are the other limitations of this study.

\section{Conclusion}

ASD device closure can be done in patients with metabolic and genetic diseases with special attention to those diseases and the potential risks they may have for this procedure.

\section{Aknowledgement}

We wish to thank Dr Vahid Ziaee for his inspirations, helps and efforts.

\section{LEGEND}

Figure 1. Brain magnetic resonance imaging of case $7(a, b)$ : a. Axial diffusion-weighted imaging, b. Axial apparent diffusion coefficient; and brain magnetic resonance angiography of case 7 (c, d); reveal large areas of diffusion restriction around the right sylvian fissure, indicating an ischemic event in the territory of the right middle cerebral artery ( $\mathrm{a}, \mathrm{b}, \mathrm{c}, \mathrm{d}$ : white Arrow).

Figure 2. Patient 4 has a typical face of Seckel syndrome.

\section{Author contributions:}

KSZ developed the concept, gathered some data, and wrote the manuscript.

$\mathrm{MH}, \mathrm{NN}$ and RM gathered most of the data, wrote some parts of the manuscript, and revised the final version.

\section{Financial Support}

This research received no specific grant from any funding agency, commercial or not-for-profit sectors.

\section{Conflicts of Interest}

None

\section{Ethical Standards}

The authors assert that all procedures contributing to this work comply with the ethical standards of the Iranian national guidelines on human experimentation and with the Helsinki Declaration of 1975, as revised in 2008, and has been approved by the Children's Medical Center ethical committee.

\section{References}

1. King TD, Mills NL. Nonoperative closure of atrial septal defects. Surgery. 1974;75(3):383-8.

2. Zanjani KS, Zeinaloo A, Malakan-Rad E, Kiani A, Bagheri M. Transcatheter atrial septal defect closure under transthorasic echocardiography in children Iran J Pediatr. 2011;21(4):473-8.

3. Nakayama R, Takaya Y, Akagi T, Watanabe N, Miki T, Nakagawa K, et al. Efficacy and safety of atrial septal defect closure using Occlutech Figulla Flex II compared with Amplatzer Septal Occluder. Heart Vessels. 2021;36(5):704-9. 
4. Mortezaeian H, Sayadpour Zanjani K, Malakan Rad E. Transcatheter atrial septal defect closure using occlutech figulla device: a two-center experience. J Tehran Heart Cent. 2013;8(4):197-201.

5. Eshraghi A, Aghaei-Moghadam E, Zeinaloo A, Rad EM, Zanjani KS, Ghalibafan SF. Atrial septal defect device closure. Is balloon sizing necessary? Iran J Pediatr. 2021;31(2):1-6.

6. Zeinaloo AA, Meraji SM, Zanjani KS, Mirzaaghayan MR. Device occlusion versus surgery for closure of congenital heart defects: Cost issues in Iran J Teh Univ Heart Ctr. 2008;3(3):141-4.

7. Kraeva N, Zvaritch E, Frodis W, Sizova O, Kraev A, MacLennan DH, et al. CASQ1 gene is an unlikely candidate for malignant hyperthermia susceptibility in the North American population. Anesthesiology. 2013;118(2):344-9.

8. Niezgoda J, Morgan PG. Anesthetic considerations in patients with mitochondrial defects. Paediatr Anaesth. 2013;23(9):785-93.

9. Wyss Y, Quandt D, Weber R, Stiasny B, Weber B, Knirsch W, et al. Interventional Closure of Secundum Type Atrial Septal Defects in Infants Less Than 10 Kilograms: Indications and Procedural Outcome. J Interv Cardiol. 2016;29(6):646-53.

10. Bartakian S, Fagan TE, Schaffer MS, Darst JR. Device closure of secundum atrial septal defects in children $<15 \mathrm{~kg}$ : complication rates and indications for referral. JACC Cardiovasc Interv. 2012;5(11):117884 .

11. Houeijeh A, Hascoët S, Bouvaist H, Hadeed K, Petit J, Godart F, et al. Transcatheter closure of large atrial septal defects (ASDs) in symptomatic children with device/weight ratio [?]1.5. Int J Cardiol. 2018;267:84-7.

12. Sobczak AIS, Stewart AJ. Coagulatory Defects in Type-1 and Type-2 Diabetes. Int J Mol Sci. $2019 ; 20(24)$.

13. Yeoh C, Teng H, Jackson J, Hingula L, Irie T, Legler A, et al. Metabolic Disorders and Anesthesia. Curr Anesthesiol Rep. 2019;9(3):340-59.

14. von Ungern-Sternberg BS, Habre W. Pediatric anesthesia-potential risks and their assessment: part II. Paediatr Anaesth. 2007;17(4):311-20.

15. Von Ungern-Sternberg BS, Habre W. Pediatric anesthesia - potential risks and their assessment: part I. Paediatr Anaesth. 2007;17(3):206-15.

16. Hanley PJ, Ray J, Brandt U, Daut J. Halothane, isoflurane and sevoflurane inhibit NADH:ubiquinone oxidoreductase (complex I) of cardiac mitochondria. J Physiol. 2002;544(3):687-93.

17. Hsieh VC, Krane EJ, Morgan PG. Mitochondrial aisease and anesthesia. J Inborn Errors Metab Screen. 2017;5(1):1-5.

18. Purza R, Ghosh S, Walker C, Hiebert B, Koley L, Mackenzie GS, et al. Transesophageal Echocardiography Complications in Adult Cardiac Surgery: A Retrospective Cohort Study. Ann Thorac Surg. 2017;103(3):795-802.

19. Doğan V, Senocak F, Orün UA, Ceylan O. Heart failure after transvenous closure of atrial septal defect associated with atrial standstill and thiamine-responsive megaloblastic anemia. Turk Kardiyol Dern Ars. $2013 ; 41(7): 638-41$.

20. Saeidi M, Shahbandari M. A Child with Seckel Syndrome and Arterial Stenosis: Case Report and Literature Review. Int Med Case Rep J. 2020;13:159-63.

21. Kernohan KD, Dyment DA, Pupavac M, Cramer Z, McBride A, Bernard G, et al. Matchmaking facilitates the diagnosis of an autosomal-recessive mitochondrial disease caused by biallelic mutation of the tRNA isopentenyltransferase (TRIT1) gene. Hum Mutat. 2017;38(5):511-6. 
22. Sharma AD, Erb T, Schulman SR, Sreeram G, Slaughter TF. Anaesthetic considerations for a child with combined Prader-Willi syndrome and mitochondrial myopathy. Paediatr Anaesth. 2001;11(4):488-90.

23. Harris M, Cao QL, Waight D, Hijazi ZM. Successful combined orthotopic liver transplant and transcatheter management of atrial septal defect, patent ductus arteriosus, and peripheral pulmonic stenosis in a small infant with Alagille syndrome. Pediatr Cardiol. 2002;23(6):650-4.
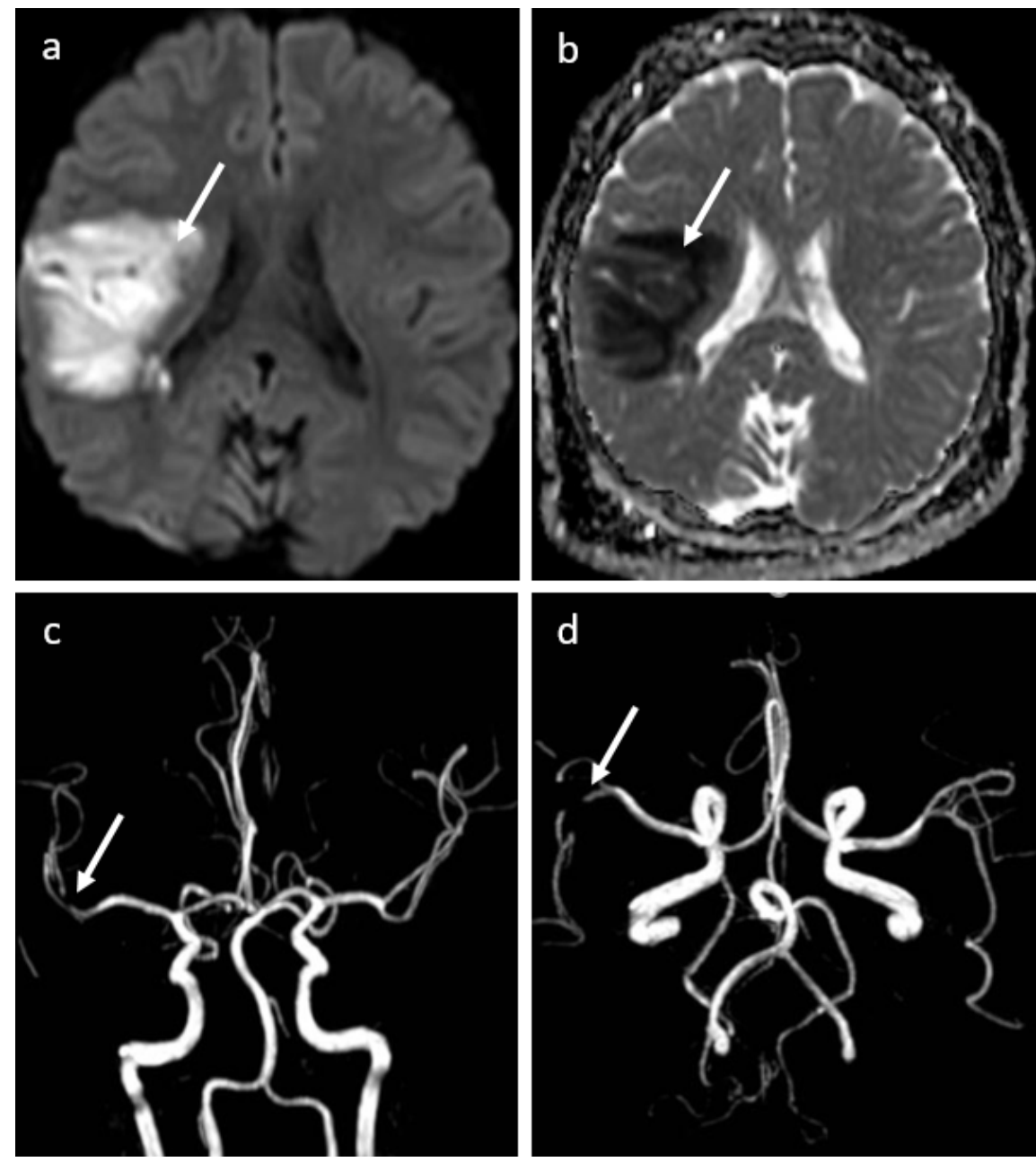


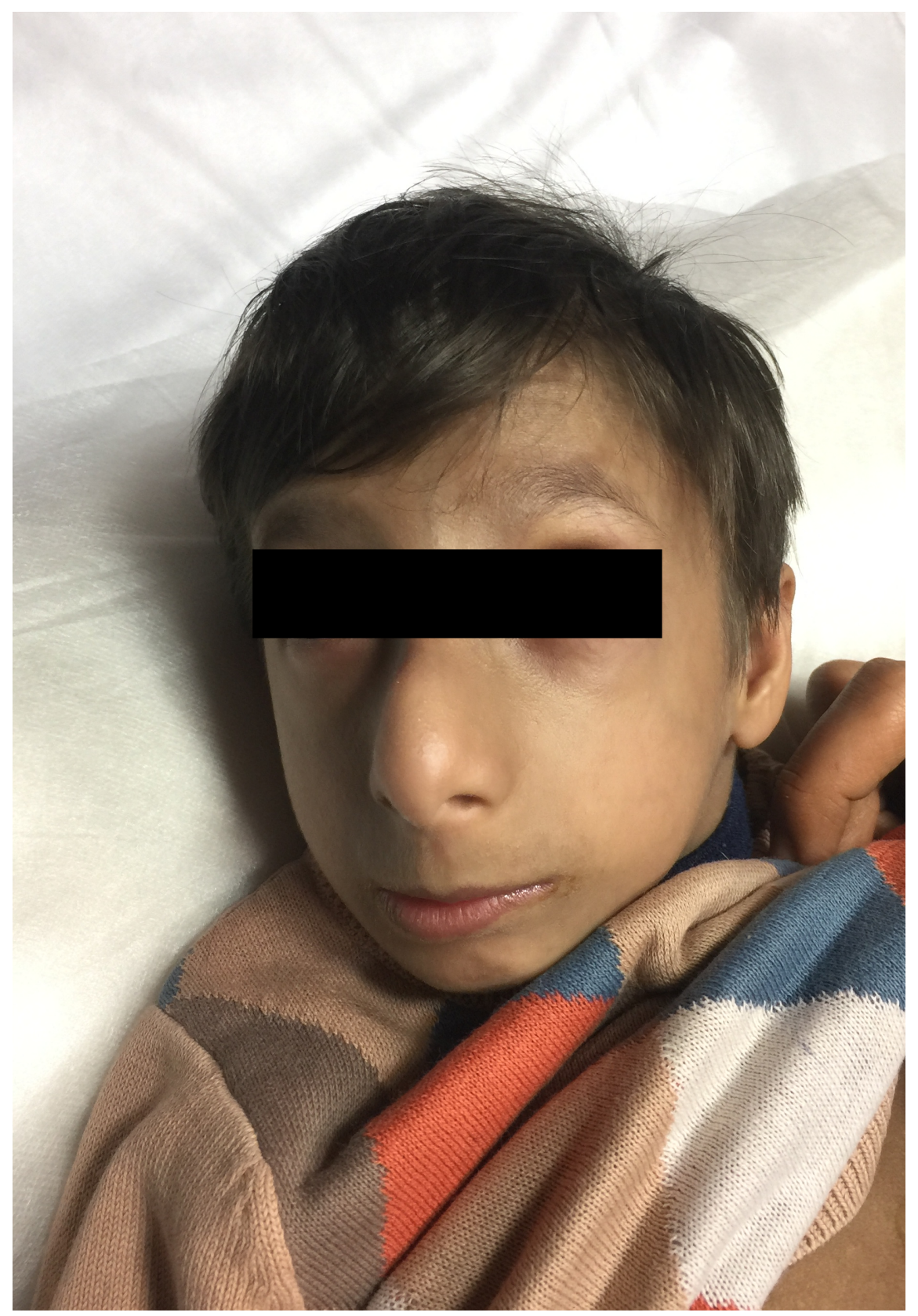

